\title{
THE NEW UNDULATOR BASED fS-SLICING BEAMLINE AT THE ALS*
}

\author{
C. Steier ${ }^{\dagger}$, D. Robin, F. Sannibale, R. Schoenlein, W. Wan, W. Wittmer, A. Zholents \\ Lawrence Berkeley National Laboratory, Berkeley, CA 94720, USA
}

\section{Abstract}

The Femtoslicing beamline at the ALS employs a fs laser beam interacting resonantly with the electron beam in a wiggler. The induced energy spread over the fs duration is converted to a transverse displacement by exploiting the storage ring dispersion. The displaced fs pulse radiates and produces fs synchrotron radiation. Up to now a regular bending magnet was used as radiator. To improve the flux, a significant upgrade was implemented, replacing the modulator, installing an in-vacuum undulator as new radiator, and installing a higher repeptition rate laser system. The new beamline will provide 100-200 fs long pulses of soft and hard x-rays with a repetition rate of $10-40 \mathrm{kHz}$ for experiments concerning ultrafast dynamics in solid state physics, chemistry and biology.

\section{INTRODUCTION}

The ALS is a third generation synchrotron light source located at Lawrence Berkeley National Laboratory. To generate short pulses of $\mathrm{x}$-rays with durations of a few hundred femtoseconds, an innovative technique has been developed [1]. This technique uses the interaction of an electron bunch with a femtosecond laser beam within an undulator to energy-modulate (slice) a short section of that bunch. Using spatial or angular dispersion downstream of the interaction with the laser one can then isolate fs x-ray pulses. Over the past years initial scientific experiments have been carried out using an ALS bending magnet beamline. Based on the performance limitations of that beamline, an upgrade as been started, which will enable more experiments that make use of time resolved spectroscopic techniques (time resolved $\mathrm{x}$-ray diffraction or time resolved $\mathrm{x}$-ray absorption spectroscopy) and require higher average photon flux than can be delivered by the bending magnet beamline. A sketch of the main accelerator components of the new beamline is shown in Fig. 1. Other light sources like BESSY and SLS have started as well to implement undulator beamlines based on similar concepts.

The upgrade consists of two new insertion devices (modulator and radiator), two new undulator beamlines, a new laser system with significantly higher repetition rate and modifications to the storage ring to create the vertical dispersion bump used to spatially separate the sliced electrons/photons. One of the new insertion devices installed in 2004 is a wiggler which is simultaneously used for protein crystallography and as modulator for the slicing. It allows optimized use both by the protein crystallographers

\footnotetext{
* This work was supported by the U.S. Department of Energy, under Contract No. DE-AC03-76SF00098.

$\dagger$ CSteier@1bl.gov
}

and the slicing experiments by allowing slicing on its first instead of third harmonic. The second insertion device installed in 2005 is an in-vacuum undulator. Since the photon energy range required by the science case is very wide (about $200 \mathrm{eV}$ to $10 \mathrm{keV}$ ), this undulator will be used as an undulator up to about $4 \mathrm{keV}$ and as a wiggler up to $10 \mathrm{keV}$.

\section{CHALLENGeS}

The accelerator physics efforts to support the new fs $\mathrm{x}$ ray undulator beamline have been centered on three main areas: generating the vertical dispersion bump to provide the spatial separation to isolate the fs $\mathrm{x}$-rays pulses, minimizing the vertical emittance and spurious dispersion, and studying insertion device related issues $[2,3,4]$. The issues studied in connection with the insertion devices include to just name some examples - their effect on the nonlinear dynamics, resistive wall heating effects, impedance issues and the effects caused by the field imperfections of the devices (coupling, focusing and orbit errors). Working together with the magnet group of the ALS, detailed specifications were developed for both devices. The wiggler has been build in house, whereas the in-vacuum undulator was build by Neomax, with significant involvement of ALS engineering in design modifications, quality control and magnetic measurements.

\section{PROJECT STATUS}

Over the last 3 years, the upgrade of the fs-slicing facility has been nearly completed and the commissioning is now starting. The new wiggler was commissioned in 2004. After initially demonstrating a simple local dispersion bump in 2002 and later studying the momentum aperture in the ALS for vertical physical apertures as small as $5 \mathrm{~mm}$, the interaction of the dispersion bump and the small apertures were studied in the second half of 2003 and no significant problems were found. A new improved lattice of the ALS implemented in 2004 required a modification of the initial skew quadrupole scheme. Initial tests of this more complicated scheme were successfully carried out in 2005 . All skew qudrupole modifications necessary were finished in April 2005. The new in-vacuum insertion device together with new vacuum chambers, beam diagnostics, chicane magnets, interlock systems, and beam aperture defining collimator were installed as well in April 2005. The commissioning of the complete system is now underway. However, the beamlines and new laser system are not quite finished. The $20 \mathrm{kHz}$ laser system is partly assembled and now being commissioned. The plan is to have it operational by July 2005 . The soft $\mathrm{x}$-ray branchline is being assembled 


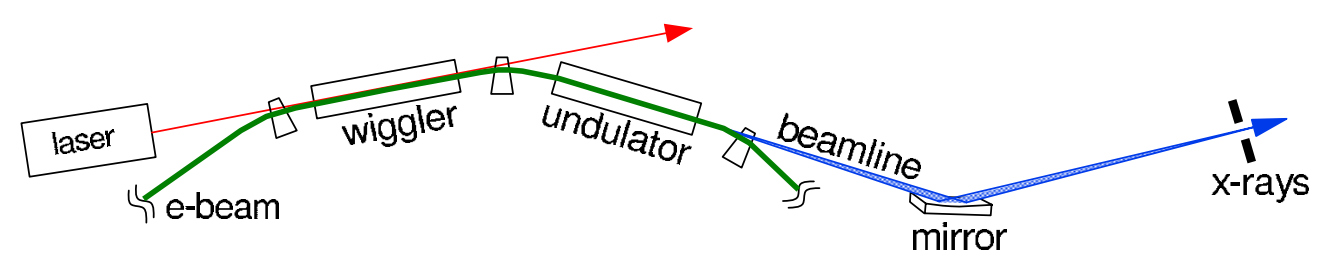

Figure 1: Sketch of slicing arrangement at ALS.

as well and will be ready to receive first light by August. The hard x-ray branchline will be installed in early 2006.

\section{NEW INSERTION DEVICES}

As the first insertion device for the upgrade, the new wiggler $(1.9 \mathrm{~T}, 114 \mathrm{~mm})$ was installed last year. One original area of concern was the potential impact of the new insertion devices on the beam dynamics [5], especially of the wiggler. The possible effects (compare [6]) were studied extensively both for the new wiggler as for the in-vacuum undulator in simulations before finishing their magnetic designs. Symplectic integrators were used to model the insertion devices [7] as well as high order taylor maps generated with runge-kutta integrators. In both cases $3 \mathrm{~d}$ magnetic field data from TOSCA was used. For the wiggler, the geometry of the magnetic assembly was chosen such that the nonlinear effects are negligible. Once the device was installed this was verified experimentally.

The protein crystallography beamline using the wiggler employs carbon filters in the beamline frontend, to reduce the heat load on the beamline optics and especially the beryllium window significantly. As part of the upgrade to the new shorter period wiggler, which delivers a higher power density, a complete redesign of those carbon filters was carried out. The old carbon filters used many layers of polycrystalline carbon which were radiatively cooled. Main disadvantages of this concept were the relatively slow initial scrubbing progress, requiring significant beamtime in order to achieve satisfactory vacuum perssures, and the very high operating temperatures, leading to quick loss of carbon due to sublimation. Because of that, the filters had to be replaced about once a year. The new carbon filter design uses only two layers of highly oriented carbon, clamped between watercooled metal frames. The new filters are now in use for about a year and a half during which they have performed flawlessly. Because of the much lower operating temperatures no material loss has been observed so far. Necessary scrubbing times have been minimized two a few hours even after the initial installation.

The in vacuum undulator was installed in the ALS in April 2005. It is the first such device installed in the ALS and has a minimum physical aperture of about $5.2 \mathrm{~mm}$, compared to the about $8 \mathrm{~mm}$ of the smallest physical aperture so far. Extensive dynamics studies have been carried out beforehand, to demonstrate that this reduction in physical aperture will not have a significant impact on the momentum aperture [8]. The initial commissioning of the device is underway and no mayor surprises have been found so far. The undulator was build in industry (Neomax). It contains some design changes compared to the earlier ones (for Spring-8, SLS, NSLS) and LBNL engineering was closely involved in design, magnetic measurements, and quality control. The undulator has a period of $30 \mathrm{~mm}$, is a hybrid type with 50 periods, a $5.5 \mathrm{~mm}$ min. magnetic gap and $1.52 \mathrm{~T}$ peak field. Fig. 2 shows a picture of the final assembly at Neomax.

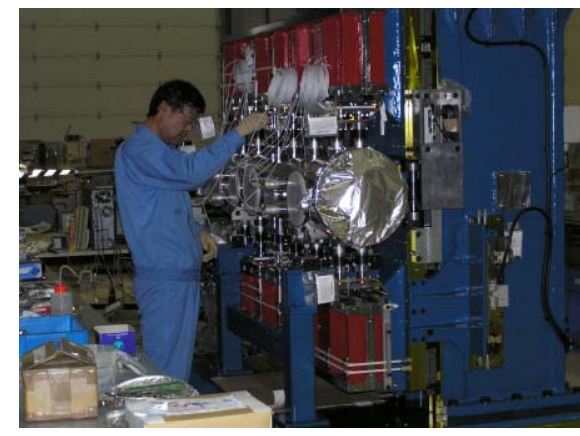

Figure 2: Picture of the first ALS in-vacuum undulator with $3 \mathrm{~cm}$ period and $1.52 \mathrm{~T}$ maximum field being assembled at Neomax.

\section{BEAM DIAGNOSTICS}

The fs-slicing project is a perfect example, where originally unrelated fundamental accelerator physics studies resulted in a significant improvement in the performance of an experiment. As part of the studies of coherent $\mathrm{THz}$ radiation at the ALS which were aimed at establishing the feasibility of a dedicated storage ring source, the emmission of radiation by the sliced bunch was studied in detail. It turns out that the slicing creates a hole at the center of the bunch distribution and the sharp edges of this hole radiate significant amounts of coherent synchrotron radiation. The intensity and spectrum of this radiation can be used to study the details of the laser interaction with the electron bunch. This is a very good non-destructive diagnostics to optimize the laser-beam interaction. It turned out to be the major breakthrough in making experiments on the original bend magnet slicing source successful. It enabled online adjustment of the laser beam, compensating for drifts in the laser pointing stability. This form of diagnostics, first demonstrated at the ALS, is now used routinely as well at BESSY.

\section{COLLIMATION SYSTEM}

One major issue with permanent magnet insertion devices, especially when operating with relatively short life- 
times in top-off mode is radiation damage. Though damage so far has mostly been observed for undulators with small $H_{c i}$ and not heat-treated material and top-off is not planned to start at the ALS before 2006, we decided to take precautionary measures. As part of the top-off upgrade, beam loss patterns for injection, stored beam losses, as well as beam trips were studied in detail. A heat treated, high $H_{c i}$ $\mathrm{NdFeB}$ material was used (Neomax-38VH). Realtime radiation monitors were installed around the undulator and an adjustable vertical collimator was designed. The first of those beam aperture deining collimators was installed at the sam etime as the undulator in a straight without insertion devices or user beamlines. It will both locate stored beam losses away from people and sensitive equipment as well as prevent injection mishaps when the undulator in the future will be closed during top-off injection. Fig. 3 shows a CAD drawing of the movable collimator.

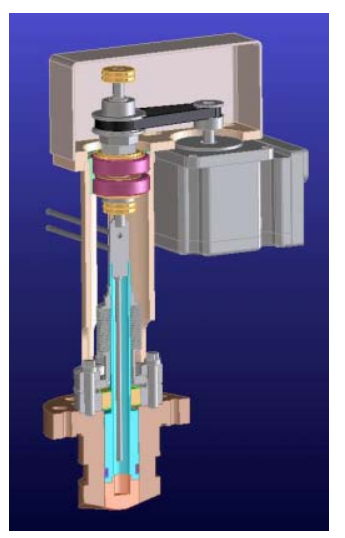

Figure 3: Beam aperture defining collimator used to locate beam losses away from the in-vacuum undulator.

\section{NEW VERTICAL DISPERSION BUMP}

The vertical dispersion bump to provide the spatial separation of the slice in the radiator was demonstrated using only 4 skew quadrupoles in 2002. This was the minimum scheme to generate a closed dispersion bump and worked because coincidentally the phase advance between the skew quadrupoles canceled coupling effects nearly perfectly. The momentum aperture with the dispersion bump was virtually unchanged. Later in 2003 it was demonstrated that even inside the $4 \mathrm{~cm}$ vertical dispersion bump a $5 \mathrm{~mm}$ vertical physical aperture was possible with negligible impact on the beam lifetime.

However, while the beamline was being build, a new improved lattice was implemented in the ALS. It features smaller beta functions in straights resulting in higher brightness, better correction of focusing effects of insertion devices and therefore larger momentum aperture. However, the phase advance between the skew quadrupoles for the dispersion bump in the new lattice changed enough that the cancellation effect with respect to coupling did not work anymore. Therefore a modification of the skew quadrupole scheme was required: Now 12 (instead of 4) skew quadrupoles are used, spanning 3 (instead of 2) arcs. Even though the new scheme has much higher complexity, it actually has many advantages over the old scheme. The most relevant is the fact that it provides improvements in terms of the single particle beam dynamics. The coupling is not just zero everywhere outside of the bump, in addition the local coupling in the straight with the in vacuum undulator is nearly zero. Fig. 4 shows the lattice functions as well as the cpupling angles for the new skew quadrupole scheme.
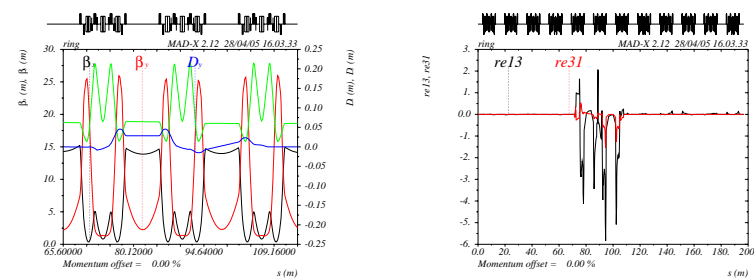

Figure 4: Lattice functions and coupling angles for the new dispersion bump using 12 instead of 4 skew quadrupoles. Global coupling as well as local coupling in the in-vacuum undulator straight are very small.

\section{SUMMARY}

A new undulator beamline for femtosecond $\mathrm{x}$-rays is being commissioned at the ALS. The accelerator physics issues associated with the new facility have been studied successfully. The dynamics in the insertion devices has been studied extensively and no problems were found. A vertical dispersion bump for spatial separation of the fs $\mathrm{x}$-rays has been demonstrated with more than sufficient amplitude and minimal negative impact. Methods for vertical emittance and dispersion control have been established, achieving better results than necessary. The impact of small gaps on lifetime and injection efficiency have been studied with positive results. Both new insertion devices required for the project have been installed. The final commissioning of the complete system is now underway.

\section{REFERENCES}

[1] Zholents and Zolotorev, Phys. Rev. Lett., 76, 916,1996.

[2] C. Steier et al., Coupling correction and Beam Dynamics at Ultralow Vertical Emittance in the ALS, Proceedings of PAC 2003, Portland.

[3] W. Wan et al., ID Modeling at the ALS, Proceedings of PAC 2003, Portland.

[4] D. Robin et al., Impact of Narrow Gap Chambers on the Lifetime at the ALS, Proceedings of PAC 2003, Portland.

[5] C. Steier et al., Phys. Rev. E 65, 056506 (2002).

[6] J. Safranek et al., Phys. Rev. STAB 5, 010701 (2002).

[7] Y. Wu et al., Proceedings of PAC 2001, Chicago, USA, 459 (2001).

[8] C. Steier, et al., Accelerator Physics Challenges of the fsSlicing Upgrade at the ALS, Proceedings of PAC 2003, Portland. 